\title{
VALUE ADDITION TO LOCALLY PRODUCED SOYBEANS IN GHANA: PRODUCTION OF SOY SAUCE USING STARTER CULTURE FERMENTATION
}

\author{
V. P. Dzogbefia, P. L. Arthur and H. D. Zakpaa \\ Department of Biochemistry and Biotechnology, \\ Kwame Nkrumah University of Science \& Technology, \\ University Post Office Kumasi, Ghana.
}

\begin{abstract}
Ghana produces about 50,000 metric tons of soy beans per annum, of which only about 15 metric tons are utilized. One aspect of utilizing the beans is in the production of soy sauce, a product whose demand is on the increase due to changing food habits of the Ghanaian society. A preliminary attempt to produce soy sauce from the cultivated beans using starter culture fermentation approach was therefore investigated. A mixture of cooked soybean mash and roasted wheat was inoculated with Aspergillus oryzae (ATCC 46240) under aseptic conditions and allowed to ferment for five days. This fermented solid mash referred to as "koji" was then mixed with an eighteen per cent brine solution and subjected to further fermentation for three months to constitute the "moromi" stage of soy sauce production. At the end of fermentation, the "moromi" was filtered and coloured with either caramel or molasses. Chemical and sensory analysis on the resultant product showed it to be comparable and in some instances superior to commercial products on the market. This simple biotechnological method could be used to add value to surplus soy beans on the Ghanaian market.
\end{abstract}

Keywords: starter culture fermentation; biotechnological method; value addition; koji; moromi; soy sauce.

\section{INTRODUCTION}

Soy sauce is a fermentation product obtained from soy beans (Glycine $\max \mathrm{L}$ ) and wheat. It is used as flavouring and seasoning in dishes. It is amino acid-rich, mildly alcoholic with great nutritional and health benefits (Peppler, 1967; Shin et al., 1995; Shon et al,. 1996; Kim, 1995).
In recent times, soy sauce has gained popularity in Ghana due to the tremendous change in the food consumption patterns and the taste for foreign foods. In the Kumasi metropolis, soy sauce is widely used especially in the preparation of fried rice. Figures available showed that the importation of soy sauce into the country will continue to increase (Ministry of Trade and Industry,

22 Journal of Science and Technology, Volume 27 no. 2, August, 2007 
2002). The major raw material for soy sauce production, the soy bean, is however cultivated in Ghana but highly under - utilized. Out of the 50,000 metric tons of soy beans produced annually, only about 15 metric tons are utilized (Ghana Export Promotion Council, 2002) while the remainder is woefully under-utilised. The low patronage of the beans is due to the long cooking time, its un-palatability and presence of anti-nutritional factors such as trypsin inhibitor and phytoestrogen (Plahar, 2003; Steinkraus, 1964). One way by which anti-nutrients and other unwanted attributes of the bean could be removed to increase its utilisation is through fermentation with moulds such as Aspergillus oryzae into soy sauce (Lee et al., 1999). The objective of this report therefore was to use starter culture fermentation approach for the production of soy sauce from locally cultivated soy beans in order to add value to this agricultural produce. The starter culture method of soy sauce production was adopted instead of the widely used chemical hydrolysis of soybean proteins (Aehle, 2004; Kikkoman, 2004; Luh, 1995) since some soy sauces produced by chemical hydrolysis have been shown to contain chemicals that are carcinogenic and therefore injurious to the health of the consumer of such products (www.foodstandards.gov.uk/saferating; www. nzherald.co.nz., ; 10/01/06).

\section{MATERIALS AND METHODS \\ Materials}

Soybeans (Anidaso variety) was obtained from the Grains and Legumes Development Board of Ministry of Food and Agriculture Kumasi, Ghana while wheat was purchased from the central market, Kejetia in Kumasi. Aspergillus oryzae (ATCC 46249) was obtained from American Type Culture Collection, Manassas, Virginia, USA.

\section{Methodology}

Soybeans were cleaned by removal of extraneous material and $0.2 \mathrm{Kg}$ milled using CYCLOTEC, 1093 mill into a powder and its proximate com- position determined before use. The starter culture Aspergillus oryzae (ATCC 46249) was propagated $(72 \mathrm{~h})$ in malt extract agar which was sterilized at $121^{\circ} \mathrm{C}$ for $15 \mathrm{~min}$, prior to its use for inoculation.

Soy beans $(1 \mathrm{Kg})$ were soaked in water at room temperature for $15 \mathrm{hr}$ after which they were poured into a stainless canister and autoclaved at $121^{\circ} \mathrm{C}$ for $1 \mathrm{hr}$. The beans were then cooled and blended with a warring blender (WARRING commercial blender, USA). The wheat was also roasted (ARISTON electric cooker, Italy) at $180^{\circ} \mathrm{C}$ for $20 \mathrm{~min}$, cooled and crushed into approximately 5 pieces per grain. The mashed soybeans and roasted wheat were mixed in a ratio of $54 \%$ : $46 \%$ ( $1 \mathrm{~kg}$ soybeans to $0.85 \mathrm{~kg}$ wheat). The mixture was spread on an alunminium tray and inoculated with Aspergillus oryzae ATCC 46249 and incubated at room temperature. The mixture was turned once on the third day and became overgrown with the mould by the fifth day. This solid mass constitutes the "koji" stage of soy sauce production. Control samples were not inoculated with culture. The experiment was conducted in triplicate.

\section{Brine fermentation (moromi)}

The koji obtained above was mixed with $5 \mathrm{~L}$ of $18 \%$ brine solution in plastic containers. The containers were covered with the lid and the mixture allowed to undergo fermentation for three months while monitoring the temperature and the $\mathrm{pH}$. The $\mathrm{pH}$ was monitored using an accurate model OSK 11473 Analogue/Digital pH meter (Ogawa Seiki Co. Ltd. Japan) equipped with an electrode and temperature control system. At the end of the fermentation, the moromi was poured into a white clean polyester cloth and pressed to drain off the liquid (shoyu) while discarding the soybean-wheat meal. This liquid is the soy sauce, normally referred to as shoyu. This was stored to age for one month. The shoyu was clarified with alum to remove heat-coagulated proteins. The shoyu was then mixed with either caramel or crude molasses to impart color and enhance the 
Figure 1 shows the flow chart for the production process of soy sauce
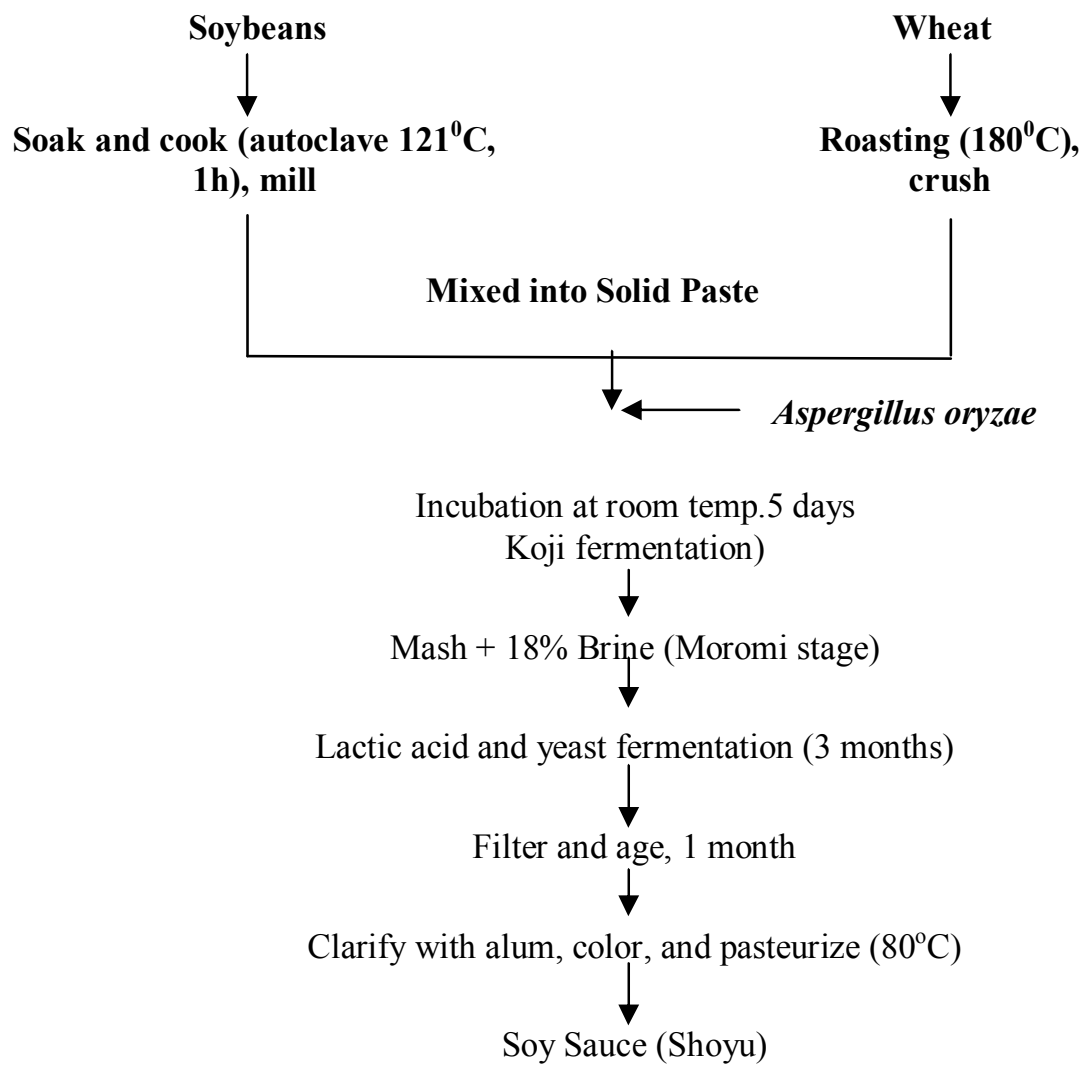

Fig.1: Flow chart for soy sauce production using Aspergillus oryzae as starter culture

taste. The ratio of either caramel or molasses to shoyu was $1 \mathrm{~g}$ or $2 \mathrm{~g}$ to $5 \mathrm{ml}$ shoyu for each colourant. The resultant product, after coloring, was pasteurized at $80^{\circ} \mathrm{C}$ for $15 \mathrm{~min}$ and bottled to constitute the final product.

The final product was subjected to physicochemical analysis and sensory evaluation to ascertain its acceptability or otherwise. Protein was analysed

Amino acids were identified by one way paper chromatography in $n$-butanol-acetic acid-water (4:1:1v/v) using amino acid standards which were prepared in $10 \%$ isopropanol at a concentration of $0.02 \mathrm{M}$. Soy sauce samples were also prepared in the same solvent. The chromatogram was run until the solvent front was $5 \mathrm{~cm}$ from the top. After drying the paper in a fume chamber, it was sprayed with Ninhydrin reagent in acetone. The paper was then dried in the oven at $110^{\circ} \mathrm{C}$ for $5 \mathrm{~min}$. and the colored spots marked. The retention factors $\left(\mathrm{R}_{\mathrm{f}}\right)$ were computed and used to identify the individual amino acids using the $R_{f}$ values of the standard amino acids run concur-

24 Journal of Science and Technology, Volume 27 no. 2, August, 2007 
rently. Ethanol was determined with PERKIN ELMER Auto System XL Gas Chromatograph, using Flame Ionisation Detector. Ethanol (Analar grade) was used to prepare standards of 2, 5, and $10 \%$ (vol./vol.) in distilled water. These standards were used to calibrate the gas Chromatograph. Total soluble solids were determined with a hand refractometer (CARL ZEISS 121554, Germany). Protein content of the soy sauce was determined by Kjeldahl digestion method (AOAC, 1990).

\section{Sensory evaluation}

Sensory evaluation, using 24 member trained panellists, was carried out by providing both the raw soy sauce and "fried rice" prepared with the soy sauce products and panellists were asked to score the different attributes on a seven-point hedonic scale. The overall acceptability of the product combining all the attributes was evaluated using a nine-point hedonic scale.

\section{Data analysis}

The sensory data generated was subjected to Kruskal-Wallis test for non-parametric data using Statistical Package for Social Scientists (SPSS Ver 11.0).

\section{RESULTS AND DISCUSSION}

Starter culture fermentation has been shown to be a positive contribution of biotechnology to the production of traditional fermented foods. The starter culture ensures the elimination of undesirable microorganisms, thus removing any danger of toxic compounds being liberated into the food. It also shortens the period required for fermentation by the traditional method. The starter culture fermentation employed enabled us to produce soy sauce in about 3 months instead of the $2-3$ years required by the traditional method (Yokotsuka, 1960).

Paper chromatography of the soy sauce revealed it contained some of the amino acids usually detected in soy sauce, including glutamic acid, the most predominant (Table 1). Nine amino acids compared to four in the controls were identified in the inoculated samples. The Aspergillus culture inoculation could therefore contribute to the production of more amino acids as many microorganisms, including fungi, are good in the synthesis of amino acids (Atasoglu et al, 1998; Atasoglu and Wallace, 2002). Amino acids constitute an important component of soy sauce. They contribute to the umami taste, act as flavour potentiators and also augment the nutritional value of soy sauce (Kikkoman, 2003). About 9 different amino acids were detected in the samples; but 17 are normally identified in soy sauce (Peppler, 1967). This difference could be due to the paper chromatography used, which was not sensitive enough to detect those amino acids which were present in very minute quantities. The starter culture fermentation took only 13 weeks to complete, but it is however known that the longer the fermentation, the higher the conversion of protein into amino acids and other flavourful and aromatic compounds (Kikkoman, 2004). Therefore the lower number of amino acids detected could also be attributed to this.

Table 1: Types of amino acids identified in soy sauce produced by starter culture fermentation

\begin{tabular}{lcc}
\hline \multicolumn{1}{c}{ Amino acid } & $\begin{array}{c}\text { Inoculated } \\
\text { samples }\end{array}$ & $\begin{array}{c}\text { Controls } \\
\text { (un-inoculated) }\end{array}$ \\
\hline Aspartic acid & + & + \\
Arginine & + & - \\
Lysine & + & - \\
Leucine & + & + \\
Proline & + & + \\
Glutamic acid & + & - \\
Methionine & + & - \\
Valine & + & + \\
Phenylalanine & + & - \\
\hline \multicolumn{1}{c}{$(+)$ detected } & & $(-)$ not detected
\end{tabular}

(-)not detected 
Table 2 shows some of the physico - chemical parameters determined on the finished product. Ethanol, an important component of soy sauce was also detected, albeit in a lower concentration compared to literature values of $1-2 \% \mathrm{v} / \mathrm{v}$ (Peppler, 1967). Ethanol imparts flavour and aroma to soy sauce by combining with some of the organic acids produced during fermentation to form esters and other aromatic compounds (Kikkoman, 2004). The pH and total solids of the finished product however fell within the literature values (Kikkoman, 2004) and also comparable to those of the commercial samples tested (Table 2). The $\mathrm{pH}$ contributes to the sharp, pungent smell associated with soy sauce (Kikkoman, 2003).

The protein content of the inoculated samples was significantly different from that of the uninoculated controls $(\mathrm{P}<0.05)$ which was attributed to the absence of starter culture in the controls at the koji stage of soy sauce production.

\section{Sensory characteristics of soy sauce}

The major sensory attributes used in characterizing soy sauce are the color, taste (saltiness, pungency, umami) and flavour/odour. Statistical analysis of panellists' response to color indicated no significant difference $(\mathrm{P}>0.05)$ between caramel and molasses treated samples compared with the commercial samples used as standards, al- though the caramel treated samples appeared to be closer in color to the commercial samples (Fig. 2). Thus it appeared that caramel may be a better colorant than molasses.

The typical burnt, smoky/roasty flavour of soy sauce (Hamano, 1994) was found to be present in the finished products. The panellists' reponses showed no significant difference between the starter culture fermented products and the commercial ones (Fig. 3). The flavour adds pleasant odour to the food and this is the key attribute that makes soy sauce a popular food condiment. The flavour is achieved through a series of complex chemical reactions involving ethanol, organic acids, amino acids and sugars during and after the fermentation process. All the above chemical components, including the sugars (data not shown) were detected in the finished products. The heat of pasteurization also enhances the flavour (Hamano, 1994; Kikkoman 2004)

The starter culture fermented soy sauce was found to exhibit the sharp, pungent and umami tastes as judged by the panellists with no significant difference $\boldsymbol{e n}$ ? compared with the commercial samples (Figs. $4-5$ ). The pungency is attributed to the presence of organic acids such as lactic acid, succinic acid etc. produced during the fermentation. The presence of the acids was reflected in the $\mathrm{pH}$ of the finished product (Table 2). On the contrary, the umami taste which is

Table 2: Physico-chemical composition of soy sauce produced by starter culture fermentation

\begin{tabular}{lcccccc}
\hline Sample type & $\begin{array}{c}\text { Protein } \\
\mathbf{( \% )}\end{array}$ & $\begin{array}{c}\text { Ethanol } \\
\mathbf{( \% )}\end{array}$ & Initial pH & $\begin{array}{c}\text { pH at } \\
\text { maturation }\end{array}$ & $\begin{array}{c}\text { Total solids (\%) } \\
\text { Caramel } \\
\text { Treated }\end{array}$ & $\begin{array}{c}\text { Molasses } \\
\text { Treated }\end{array}$ \\
\hline $\begin{array}{l}\text { Amoy/Chain } \mathrm{Ku} \\
\text { (imported }\end{array}$ & N.D & N.D & N.D & $4.75 / 4.80$ & 30.4 & 35.8 \\
$\begin{array}{l}\text { samples) } \\
\text { Inoculated }\end{array}$ & $4.28 \pm 0.07$ & 0.639 & $4.20 \pm 0.01$ & $4.69 \pm 0.01$ & $33.41 \pm 0.14$ & $22.34 \pm 0.05$ \\
$\begin{array}{l}\text { Un-inoculated } \\
\text { (control) }\end{array}$ & $1.86 \pm 0.04$ & 0.805 & $4.55 \pm 0.03$ & $4.81 \pm 0.07$ & $35.36 \pm 0.45$ & $22.50 \pm 0.03$ \\
\hline
\end{tabular}

Values are Averages \pm Standard Deviation $\quad$ N.D $=$ not determined

26 Journal of Science and Technology, Volume 27 no. 2, August, 2007 
translated as delicious, savoury or brothy meaty flavour is elicited by the amino acids (Kikkoman, 2004). The umami taste is a character of soy sauce that leaves a lasting spicy and aromatic taste in the mouth after tasting the sauce or meal prepared with the sauce. Although there were no significant differences among the different samples, the caramel treated samples appeared to exhibit the umami taste rather strongly compared to the molasses treated ones (Fig. 5).

The overall acceptability of the products, according to the panellists' response, did not show any significant difference among them (Fig. 6). However, one of the caramel treated samples was rated higher than even the imported commercial products. On the whole, the caramel treated samples appeared more acceptable than the molasses colored products which again confirmed the earlier observation that caramel may be a better colorant than molasses.

\section{CONCLUSION}

Starter culture fermentation approach, a more appropriate technology for a developing country as Ghana, could be adequately employed for production of soy sauce in order to add value to the soy beans produced in the country. The process can be achieved within 3 months to give an acceptable product compared to the imported products currently on the market.

\section{ACKNOWLEDGEMENT}

Aspergillus oryzae was kindly provided by Prof. Samuel Adunyah of Meharry Medical College, Nashville Tennesee, USA. The sensory evaluation data was analysed by Francis Amaglo. The authors are grateful to both.

\section{REFERENCES}

Atasoglu, C., Valdes, C., Walker, N.D., Newbold, C.J. and Wallace, R.J. (1998). De novo synthesis of amino acids by the ruminal bacteria Prevotella bryantii $\mathrm{B}_{1} 4$, Selenomonas ruminantium HD4, and Streptococcus bovis
ES1. Appl Environ. Microbiol. 64 (8): 28362843.

Atasoglu, C. and Wallace R.J. (2002). De novo synthesis of amino acids by ruminal anaerobic fungi, Piromyces communis and Neocallimastix fontalis. FEMS Microbiol. Lett. 212 (2): $243-247$.

Ghana Export Promotion Council (2002). Annual Export Performance (soy beans). General Information Department (GEPC) $\mathrm{Ku}-$ masi, Ghana. Pp. 2.

Kikkoman, Foodservice (2003). Characterising soy sauce. www.kikkoman-usa.com $(18 / 01 / 06)$

Kikkoman Foodservice (2004). Soy sauce Basics www.kikkoman-usa.com (18/01/06)

Kim, S.E. (1995). Tumor suppressive peptides derived from traditional Korean soy sauce. M. Sc. Thesis, Seoul National University, Suwon, Korea.

Lee, B. Y. Kim, D. M., and Kim, K. H. (1991). Physico-chemical properties of a viscous substance extracted from chongkook-jang. Korean J. Food Sci. Tech. 23 (5): $599-$ 604

Luh, B.S. (1995). Industrial production of soy sauce. J. Industrial Microbiol. Biotech. 14 (6): $467-471$.

Ministry of Trade \& Industry (2002). Amount of soy sauce importation into Ghana (Retrieved from Ministry of Trade and Industry Database, Import Section), Accra Ghana.

Peppler, J.H. (1967). Mould-modified foods. Microbial Technology, $2^{\text {nd }}$ Ed., Vol.2, Academic Press, New York, Pp. 94 - 97.

Plahar, W. A. (2003). Workshop on the promotion of soy beans in Ghana, Crops Research Institute $4-5^{\text {th }}$ November, 2003, Fumesua Kumasi Ghana.

Shin, Z. I., Ahn, C. W. Nam, H. S., Lee, H. J. and Moon, T. H. (1995). Fractionation of 
Angiotensin Converting Enzyme (ACE) Inhibitory Peptides from soybean paste, $\mathrm{Ko}$ rean J. Food Sc. 27 (2): 230 - 234.

Shon, D. H. Lee, K. E. Ahn, C. W. Nam, H. S. Lee, H. J. and Shin, J. K. (1996). Screening of antithrombotic peptides from soybean paste by the micro-plate method. Korean $J$. Food Sc. Tech. 28 (4): $684-688$

Soe J. B. (2004). Industrial Enzymes - flavour development. In: Enzymes in industry: Production and applications $2^{\text {nd }}$ edn. Wolfgan Aehle (Editor); Wiley - VCH Weinheim, Germany pp. 148.
Steinkraus, K. H. (1964). Pilot plant studies on soymilk. Food Technology 18: 139-168. www. foodstandards.gov.uk/safereating $(09 / 01 / 06)$ certain soy and oyster sauces containing cancer-causing chemicals www.nzherald.co.nz (09/01/06) ANZFA issues warning on consumption of soy sauce

Yokotsuka, T. (1960). Aroma and flavour of Japanese soy sauce. Adv. Food Res.10:75134 


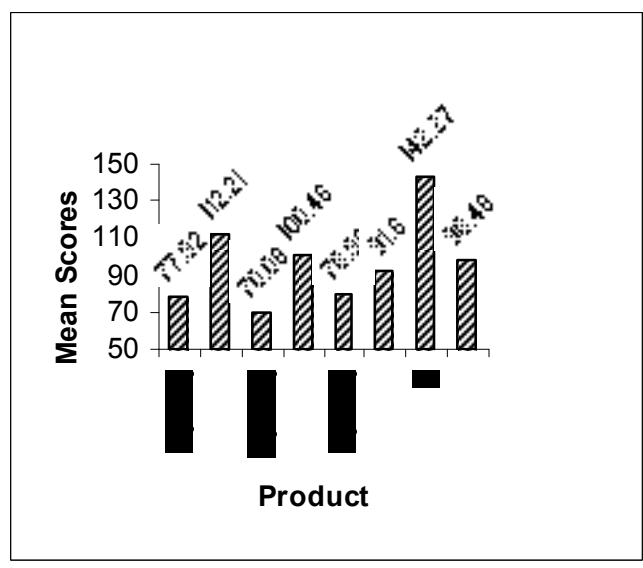

Fig. 2. Mean rank score for colour of soy sauce

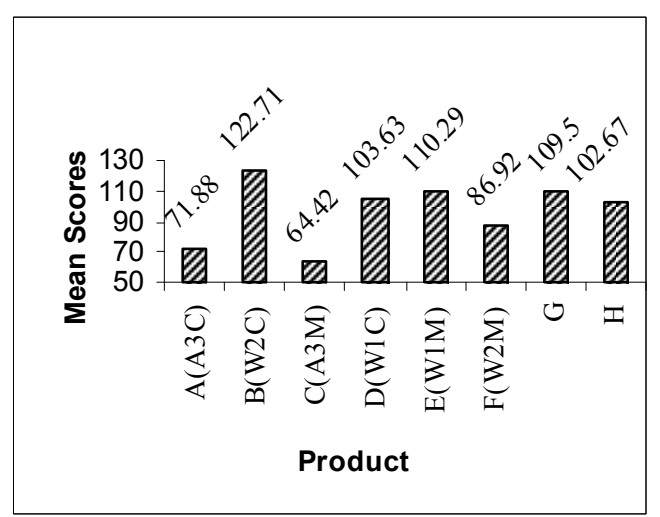

Fig.4. Mean rank scores for umami taste of soy sauce

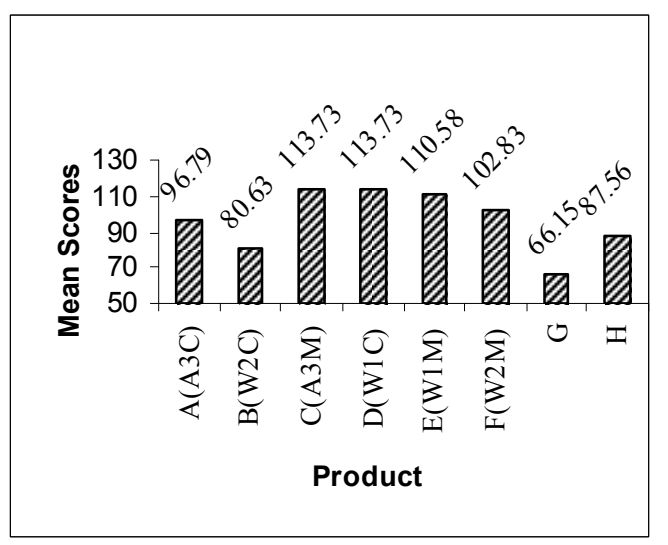

Fig.3. Mean rank scores for flavour of soy sauce

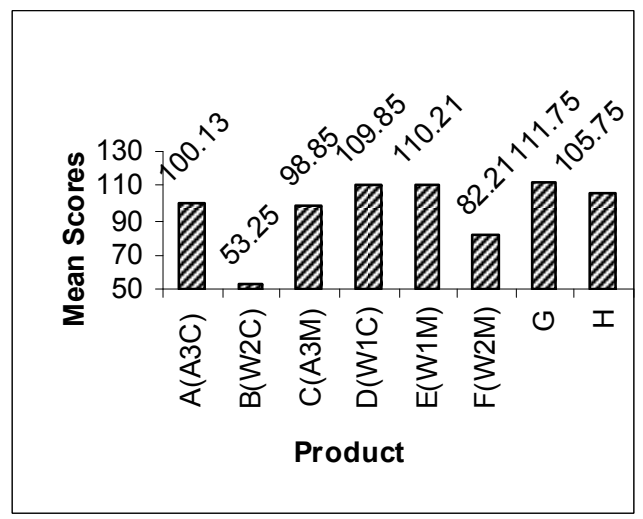

Fig.5. Mean rank scores for pungency of soy sauce

Mean ranks with the highest value correspond to the scores with the highest ranking of that attribute on the hedonic scale.

\section{Legend:}

A Control (un-inoculated) sample treated with caramel

B Starter culture inoculated sample treated with caramel

C. Control sample treated with molasses

D Replicate; starter culture inoculated sample treated with caramel

E, F Replicate starter culture inoculated samples treated with molasses

H, G Standard commercial soy sauce samples 


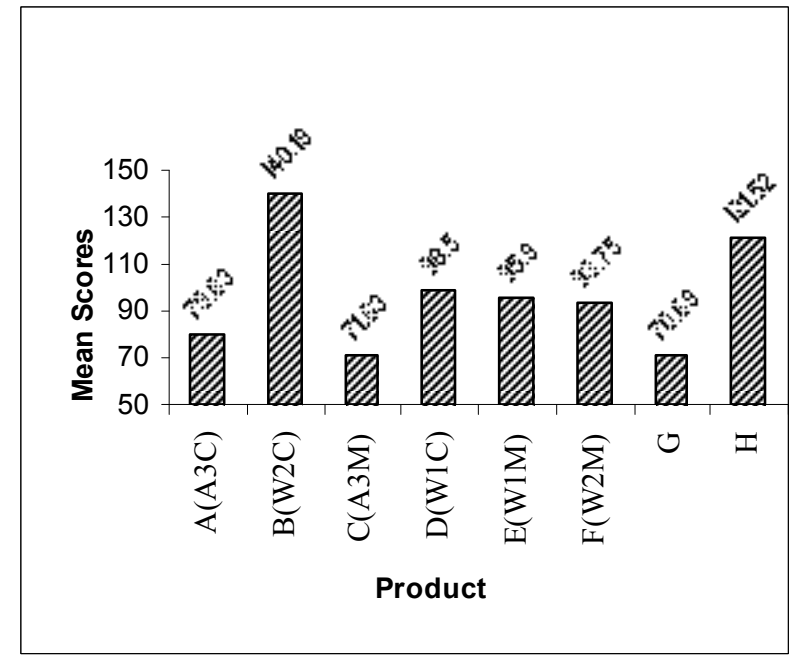

Fig. 6. Mean rank scores for the overall acceptability of soy sauce

Mean ranks with the highest value correspond to the scores with the highest ranking of that attribute on the hedonic scale.

\section{Legend:}

A Control (un-inoculated) sample treated with caramel

B Starter culture inoculated sample treated with caramel

C. Control sample treated with molasses

D Replicate; starter culture inoculated sample treated with caramel

E, F Replicate starter culture inoculated samples treated with molasses

H, G Standard commercial soy sauce samples 